\title{
USO DE LA HARINA DE KUDZU (Pueraria phaseoloides) EN RACIONES DE CERDOS EN CRECIMIENTO
}

\author{
Julio Rosales* \\ L uis García **
}

\section{RESUMEN}

La alimentación representa $70 \%$ de los costos de producción de cerdos. Una de las alternativas para disminuir costos es la utilización de insumos alimenticios no tradicionales tales como la leguminosa Pueraria phaseoloides en forma de harina. El presente estudio se realizó en el Centro Regional de Investigación del IIAP, filial U cayali. EI objetivo fue determinar los efectos biológicos y económicos de diferentes niveles de harina de kudzu en las raciones de cerdos cruzados (yorkshire $x$ landrace $x$ criollo) en crecimiento. Se trabajó con tres tratamientos: T1 ( $0 \%$ de kudzu), T2 (15\% de kudzu) y T3 (30\% de kudzu) aplicados durante 56 días. Los consumos de alimento diario ( $\mathrm{kg} /$ animal) fueron: $\mathrm{TI}=2.08 ; 12=1.61$ y T3=1.53, donde $\mathrm{T} 1$ fue mayor $(\mathrm{P}<0.05)$ al T3 (Prueba de Duncan). Esto se debería a que T3 tuvo la ración más voluminosa y menos palatable. $L$ as ganancias de peso diario promedio fueron:

$\mathrm{T} 1$ = 487; $\mathrm{T} 2=349$ y T3 = $261 \mathrm{~g} /$ animal, igualmente $\mathrm{T} 1$ fue mayor $(\mathrm{P}=0.05)$ que T3 (Prueba de Duncan), esta diferencia podría atribuirse al mayor contenido de fibra en la ración T3, lo cual afectó la digestibilidad de los nutrientes. Respecto a la conversión alimenticia el tratamiento mejor fue $\mathrm{TI}$ (4.25) con respecto a T2 (6.64) y T3 (5.85). E conómicamente el tratamiento T1 fue el que brindó mayor beneficio neto. Se concluye que el mayor consumo de alimento y mayor ganancia de peso se encontró en $\mathrm{T} 1$ respecto al T3; la mejor conversión alimenticia y beneficio económico fue el T1i en comparación al T2 y T3, respectivamente.

\section{INTRODUCCION}

La explotación de cerdos en la zona de Pucallpa es una actividad de baja rentabilidad, debido principalmente a los al tos costos de los insumos tradicionales para preparar los al imentos balanceados.

\footnotetext{
* Investigador del área Pecuaria del Centro Regional de Investigación del Instituto de Investigaciones de la A mazonía Peruana (IIAP), filial U cayali.

** Tesista de la U niversidad Nacional de U cayali.
} 
El costo de alimentación representa aproximadamente $70 \%$ de los costos directos de producción. U na de las opciones para disminuir estos costos es buscar insumos regionales no tradicionales de menor costo. En la zona contamos con leguminosas forrajeras de los géneros: Pueraria sp., Centrosema sp., Sthylosanthes sp., Desmodium sp., etc., con altos contenidos de proteína total, que ofreciéndolos directamente 0 en forma de harina, podrían reemplazar parcialmente a otros insumos proteicos tradicionales como la harina de pescado, la torta de soya y pasta de algodón. Insumos muy costosos debido al transporte desde la costa peruana.

El objetivo de este estudio fue determinar los efectos biológicos y económicos de diferentes niveles de harina de kudzu en las raciones de cerdos en crecimiento.

\section{REVISION BIBLIOGRÁFICA}

U na de las alternativas para disminuir los costos de la alimentación de cerdos es la utilización de alimentos de origen vegetal, que son menos caros que los de origen animal (Harry, 1984). Sin embargo, su utilización debe ser como sustituto parcial, debido a que las proteínas de origen vegetal tienen un valor biológico más bajo que las proteínas de origen animal. Estos tienen mayores contenidos de vitamina B12, metionina y lisina; más calcio y fósforo, y mejores niveles de otras vitaminas del complejo $B$, específicamente riboflavina (Rojas, 1982).

En la zona de Pucallpa, existen varias leguminosas promisorias; entre ellas, el kudzu (Pueraria phaseoloides) (Reyes y Ordoñez, 1985) destaca como un recurso abundante y de buen valor alimenticio. Su contenido promedio de proteína cruda es de $19.2 \%$, en base seca y $51.5 \%$ de digestibilidad in vitro de la materia seca (Ruiloba, 1990). Pero, su uso en determinadas edades del animal es limitado debido a su alto contenido de fibra cruda que varía entre el $32 \%$ (Buitrago, 1990) y el 35.7\% (Harris, 1988).

El cerdo es un animal monogástrico, que a diferencia de los rumiantes, no desarrolla procesos importantes de fermentación microbiana y este hecho limita la utilización de productos fibrosos (forrajes, bagazos, cortezas, etc.) y de nitrógeno no proteico (urea, amonio, amidas, etc.) (Buitrago, 1993).

Los alimentos fibrosos son buena fuente de energía para los rumiantes; pero su gran volumen y el tamaño limitado del tubo digestivo del cerdo restringen su uso en esta especie (Esnminger y Olentine, 1983). Por otro lado el alto contenido de fibra en la ración origina un alimento voluminoso, ocasionando menor ingesta de nutrientes digestibles (M aynard et al 1989). Concellón (1983) afirma que para evitar problemas de cerdos en crecimiento el nivel de fibra bruta no debe ser mayor a 4\%. Lucas (1986) afirma que las leguminosas en general, 
pueden incluirse en la ración en niveles de 5 a 10\% para cerdos en crecimiento y acabado, y de 15 a 35\% para marranas en gestación y lactancia. Castillo (1992), realizó un estudio en Tingo $M$ aría, utilizando raciones con niveles de harina de kudzu de $0,5,10$ y 15\% mezclado con harina de pescado, torta de soya, maíz, etc. , para cerdos en crecimiento. EI nivel adecuado de harina de kudzu fue de $5 \%$ con un consumo de alimento de $1.99 \mathrm{~kg}$ por día, consiguiente una ganancia de peso diario de $0.608 \mathrm{~kg} /$ animal, con una conversión alimenticia de 3.27 . El costo de la ración fue $0.5 \%$ menor con respecto al testigo.

\section{MATERIALES Y METODOS}

\section{UBICACION Y DURACION DEL ESTUDIO}

El estudio se realizó entre marzo y mayo de 1992, en la Estación Experimental "Villa Rica" del Centro Regional de Investigación del IIAP, filial U cayali, ubicada en el $\mathrm{Km} .12 .4$ de la carretera Federico Basadre (PucallpaLima), a 80 22' 13" S y 740 34' 23" O y a una altitud de 154 msnm.

\section{CONDICIONES CLIMATICAS}

La zona pertenece al ecosistema mayor de bosque tropical semisiempreverde estacional (Cochrane, 1982). La precipitación promedio anual es de

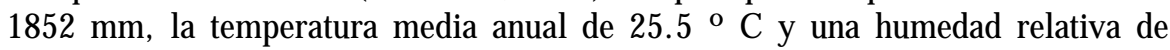
$82 \%$.

\section{INSTALACIONES Y E QUIPOS}

Se usó dos porquerizas de $24 \mathrm{~m}^{2}$ cada uno, construida sobre un estanque piscícola, con techo de hojas de palmera, piso y cerco enrejillado de madera, dividida en tres corrales de $6 \mathrm{~m}^{2}$ cada uno $(2 \times 3 \mathrm{~m})$. Cada corral fue equipado con comedero de madera y un bebedero de neumático partido por la mitad.

\section{ANIMALES}

Se usaron 18 gorrinos, nueve machos castrados y nueve hembras, cruces de las razas landrace $x$ yorkshire $x$ criollas. Los 18 gorrinos fueron bloqueados por sexo en dos grupos. Los nueve gorrinos de cada sexo fueron distribuidos al azar en tres sub-grupos de tres animales cada uno. Cada sub-grupo de animales fue criado en un corral. 


\section{TRATAMIE NTOS}

Cada grupo de animales fue alimentado con raciones isoproteícas e isoenergética, con diferentes niveles de harina de kudzu, los que originaron tres tratamientos:

$\mathrm{TI}=$ Ración con $0 \%$ de harina de kudzu

$12=$ Ración con $15 \%$ de harina de kudzu

$13=$ Ración con $30 \%$ de harina de kudzu

\section{ALIMENTACION}

Se usaron raciones de fase de crecimiento desde la 10 hasta la 18 aya semana de edad. Las raciones tuvieron $16 \%$ de proteína cruda (PC) y 3.20 $\mathrm{Mcal} / \mathrm{kg}$ de energía digestible (ED). Las fórmulas de las tres dietas experimentales y las especificaciones nutricionales se presentan en los cuadros 1 y 2. El análisis proximal de los alimentos se muestra en el cuadro 3.

\section{Cuadro 1}

Fórmula de las raciones experimentales para cerdos en fase de crecimiento

\begin{tabular}{|c|c|c|c|}
\hline \multirow{3}{*}{ Insumo } & \multicolumn{2}{|c|}{ Tratamientos } & \multirow[b]{2}{*}{ T3 } \\
\hline & T1 & $\mathrm{T} 2$ & \\
\hline & \multicolumn{3}{|c|}{ - - } \\
\hline H arina de kudzu & 0.00 & 15.00 & 30.00 \\
\hline M aíz amarillo & 50.00 & 50.00 & 50.00 \\
\hline Polvillo de arroz & 27.90 & 15.30 & 2.80 \\
\hline Hna. de pescado II & 21.10 & 18.70 & 16.20 \\
\hline Sal mineral + vitaminas & 0.65 & 0.65 & 0.65 \\
\hline Sal común & 0.35 & 0.35 & 0.35 \\
\hline Total & 100.00 & 100.00 & 100.00 \\
\hline
\end{tabular}

1/ Se utilizó el suplemento Delrromine-Vit. 


\section{Cuadro 2}

E specificaciones nutricionales calculados de las raciones experimentales para cerdos en fase de crecimiento

\begin{tabular}{llll}
\hline \multirow{2}{*}{ Nutrientes } & \multicolumn{3}{c}{ Tratamientos } \\
& T1 & T2 & T3 \\
\hline ED 1 (M cal/kg) & 3.20 & 3.21 & 3.23 \\
Proteína (\%) & 16.00 & 16.03 & 16.00 \\
Fibra (\%) & 6.47 & 6.11 & 5.76 \\
Grasa (\%) & 6.66 & 6.31 & 5.95 \\
Ca (\%) & 1.22 & 1.09 & 0.95 \\
P (\%) & 0.64 & 0.55 & 0.46 \\
\hline
\end{tabular}

1/ Energía Digestible

\section{Cuadro 3}

Análisis proximal' en base seca de las raciones para cerdos en fase de crecimiento.

\section{N utrientes}

\section{Tratamientos}

$\mathrm{Ti}$

$\mathrm{T} 2$

$\%$

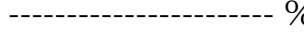

\begin{tabular}{lrrr} 
Humedad & 12.48 & 12.42 & 12.54 \\
Proteína & 15.56 & 14.26 & 14.35 \\
Grasa & 8.00 & 5.78 & 5.81 \\
Fibra & 4.54 & 6.33 & 8.51 \\
Ceniza & 11.74 & 9.79 & 10.10 \\
Nifex & 60.16 & 63.84 & 61.23 \\
\hline Total & 100.0 & 100.00 & 100.00 \\
\hline
\end{tabular}

1. L aboratorio de Suelos y T ejidos V egetales del INIA, Pucallpa

Los cerdos fueron alimentados dos veces al día; la primera a las 08:00 y la segunda a las 13:00 horas. El suministro de agua fue ad libitum teniendo como fuente la propia piscigranja. 


\section{SANIDAD}

Al inicio del experimento los animales seleccionados fueron vacunados contra cólera porcino (cepa china, virus vivo modificado), dosificados contra parásitos gastrointestinales con Levamisol - L al 7.5\% (Ripercol -) ${ }^{*}$ y bañados contra parásitos externos con hexaclorociclohexano (Sarnavet) ${ }^{* *}$

\section{VARIABLES MEDIDAS}

\section{Consumo de Alimento}

El consumo de alimento se calculó por diferencia entre el alimento ofrecido y el alimento residual y desperdicio. De esta manera se registró los consumos diario y semanal por tratamiento.

\section{Ganancia de peso}

El control de peso se realizó en forma individual al inicio y semanalmente hasta el final del experimento. Los pesos fueron tomados a las 08:00 horas con los animales en ayunas. Se usó una balanza tipo plataforma de $500 \mathrm{~kg}$ de capacidad con una aproximación de $200 \mathrm{~g}$.

\section{Conversión alimenticia}

Estuvo definido como la cantidad de alimento consumido por animal para ganar un $\mathrm{kg}$ de peso vivo.

\section{Análisis económico}

Para el análisis económico se tuvo en cuenta los costos variables y costos fijos. L os costos variables de producción fue la suma de los costos de alimentación por animal en cada tratamiento. En los costos fijos se consideraron la compra de los animales, mano de obra, medicamentos, administración, depreciación de instalaciones y equipos, e interés al capital. Los ingresos se estimaron multiplicando el precio de venta por kilo de peso vivo (PV) por el peso de cada 
animal. De la diferencia de los ingresos y el costo de producción se obtuvo la ganancia neta por animal.

La ecuación usada para el beneficio neto fue la siguiente:

$$
B=P Y i-(C V i+C F)
$$

Donde:

B $=$ Beneficio neto en nuevos soles por cerdo

$\mathrm{i}=$ Tratamientos

$\mathrm{P}=$ Precio por kilo de cerdo en nuevos soles

$\mathrm{Yi}=$ Peso promedio final por animal en $\mathrm{kg}$

$\mathrm{CVi}=$ Costo variable por cerdos en nuevos soles

$\mathrm{CF} \quad=$ Costo fijo por cerdos en nuevos soles

Diseño estadístico

Se usó el diseño estadístico Bloque Completamente Randomizado con dos repeticiones (Calzada, 1982), siendo el Modelo A ditivo Lineal

Donde:

$$
Y \mathbf{i j}=\mathbf{U}+\mathbf{t i}+\mathbf{B j}+\mathbf{E} \mathbf{j}
$$

Y ij = V alor observado para la j-ésima repetición del tratamiento i-ésimo.

$\mathrm{U} \quad=$ Media poblacional.

ti $\quad=$ Efecto del $\mathrm{j}$-ésimo tratamiento.

$\mathrm{Bi}=$ Efecto del $\mathrm{j}$-ésimo bloque, sexo.

Eij = Error experimental.

Para las comparaciones de los tratamientos se utilizó la prueba de significación de Duncan.

\section{RESULTADOS Y DISC USION}

\section{CONSUMO DE ALIMENTO}

El consumo de alimento obtenido se presenta en el cuadro 4. Las diferencias entre tratamientos fueron estadísticamente significativas $(P=0.05)$, específicamente entre T1 y T3 (Prueba de Duncan). El mayor consumo por 
animal en T1 $(2.08 \mathrm{~kg} /$ día) fue un $35.9 \%$ superior a T3 $(1.53 \mathrm{~kg} /$ día). Esto se debió posiblemente al mayor volumen de la ración del T3 que limitó una mayor ingestión del alimento ( $M$ aynard et al 1989) y además, la baja palatabilidad del kudzu en comparación con otros insumos, como el maíz, ocasionó un mayor desperdicio de la comida en el afán de seleccionar el maíz.

\section{Cuadro 4}

Consumo de alimento total y diario $(\mathrm{kg})$ de cerdos en fase de crecimiento ${ }^{1}$, durante 56 días. Promedio de dos repeticiones

\begin{tabular}{cll}
\hline Tratamientos & $\begin{array}{l}\text { Consumo } \\
\text { Total }\end{array}$ & $\begin{array}{l}\text { Consumo } \\
\text { diario }\end{array}$ \\
\hline T1 & 116.3 & $2.08 \mathrm{a}^{2}$ \\
T2 & 89.6 & $1.61 \mathrm{ab}$ \\
T3 & 85.3 & $1.53 \mathrm{~b}$ \\
\hline
\end{tabular}

1/ Cerdos de 10 semanas al inicio del experimento

$2 / \mathrm{V}$ alores seguidos por letras iguales no difieren significativamente $(\mathrm{P}=0.05)$

Consumos de alimento similares y con igual tendencia a las obtenidas en este estudio, fue reportado por Saroli (1983) en cerdos en crecimiento, los consumos diarios fueron de 2.19; 1.99; 1.94 y $1.92 \mathrm{~kg} /$ animal para los niveles de $0,5,10$ y $15 \%$ de kudzu en la ración, respectivamente.

\section{GANANCIA DE PESO}

Los pesos iniciales y finales, así como la ganancia de peso durante todo el período experimental se muestra en el cuadro 5 . Se encontraron diferencias $(P<0.06)$ en ganancia de peso entre tratamientos. A la prueba de Duncan la diferencia entre el T1 y T3 fue significativa $(P=0.05)$. En el tratamiento sin kudzu la ganancia de peso fue $86.6 \%$ superior a T3. Esta menor ganancia de peso en T3, podría atribuirse al mayor contenido de fibra cruda en la ración, lo cual determina una menor ingesta de nutrientes digestibles (M aynard et al, 1989). 


\section{Cuadro 5}

\section{Peso inicial, final y ganancia de peso $(\mathrm{kg})$ de cerdos en tase de crecimiento ${ }^{1}$, durante 56 días. Promedio de dos repeticiones}

\begin{tabular}{ccccc}
\hline Tratamiento & Peso & \multicolumn{2}{c}{ Ganancia de peso } \\
& Inicial & Final & Total & Diario \\
\hline T1 & 19.77 & 47.03 & 27.26 & $0.487 \mathrm{a}$ \\
T2 & 16.97 & 36.50 & 19.53 & $0.349 \mathrm{ab}$ \\
T3 & 16.53 & 31.14 & 14.61 & $0.261 \mathrm{~b}$ \\
\hline
\end{tabular}

$1 /$ Cerdos de 10 semanas de edad al inicio del experimento

2 ! V alores seguidos por letras iguales no difieren significativamente $(P=0.05)$

Ganancias mayores a las encontradas, pero con igual tendencia, fueron logradas por Saroli (1983). Este autor, usando 0, 5, 10 y 15\% de harina de kudzu en la ración de cerdos en crecimiento encontró aumentos de peso diarios de 677, 608, 552 y $520 \mathrm{~g} /$ animal, respectivamente. Las diferencias podrían atribuirse entre los valores encontrados por Saroli (1983) y el presente trabajo a la raza 0 cruce de animal, contenido de insumos y nutrientes en la ración y periodo de evaluación usados en cada caso.

En cerdos Large White en crecimiento, Sonaiya et al (1982) encontraron ganancias de pesos similares a las del presente estudio. A sí, con $0,15,30$ y $45 \%$ de harina de yuca en la ración, las ganancias de peso diarios fueron $0.39 ; 0.42$; 0.46

$0.43 \mathrm{~kg} /$ animal, respectivamente.

\section{Conversión alimenticia}

La conversión alimenticia durante la fase de crecimiento se muestra en el cuadro 6 , donde se encontró diferencias $(P=0.05)$ entre tratamientos. A la prueba de $D$ uncan se observó que el T1 fue el de mayor $(P=0.05)$ conversión alimenticia en un $8.9 \%$ y $37.5 \%$ con respecto a los tratamientos $\mathrm{T} 2$ y T 3 respectivamente. 


\section{Cuadro 6}

Conversión alimenticia de cerdos en fase de crecimiento ${ }^{1}$, durante 56 días. Promedio de dos repeticiones.

\begin{tabular}{llll}
\hline Tratamiento & $\begin{array}{l}\text { Consumo de } \\
\text { A limento T otal }\end{array}$ & $\begin{array}{l}\text { Ganancia de } \\
\text { Peso Total }\end{array}$ & $\begin{array}{l}\text { Conversión } \\
\text { Alimenticia }\end{array}$ \\
\hline $\mathrm{Ti}$ & 116.3 & 27.26 & $4.25 \mathrm{a}^{2}$ \\
$\mathrm{~T} 2$ & 89.6 & 19.53 & $4.64 \mathrm{~b}$ \\
$\mathrm{~T} 3$ & 85.3 & 14.61 & $5.85 \mathrm{~b}$
\end{tabular}

$1 /$ Cerdos de 10 semanas de edad al inicio del experimento

$2 /$ V alores seguidos por letras iguales no difieren significativamente $(P=0.05)$

L as menores conversiones alimenticias de T2 y T3 puede atribuirse al alto contenido de fibra de las raciones, y por tanto menor digestibilidad del alimento. Además, los alimentos fibrosos o voluminosos, por su propia conformación física, tienden a estimular el peristaltismo intestinal (Maynard, et al, 1989), acelerando la velocidad de pasaje de los alimentos en el tracto digestivo, sin que éstos sufran una buena digestión. De tal manera que los animales que consumen este tipo de alimento necesitan ingerir una mayor cantidad para formar un kilo de carne.

Los resultados de conversión alimenticia logradas fueron ligeramente menores a los reportados por Saroli (1982) y Castillo (1992). Estos encontraron conversiones de 3.70 y 4,54, respectivamente con una ración de $15 \%$ de harina de kudzu en cerdos en crecimiento. Estas diferencias podrían deberse al tipo de animal, insumos y nutrientes en la ración y período de evaluación utilizadas.

\section{ANALISIS ECONOMICO}

Para el análisis económico se usaron los costos fijos y los costos variables, tal como se indica en los cuadros 7 y 8 , respectivamente. El análisis de costos se realizó en el mes de abril de 1992, teniendo como referencia un dólar equivalente a 0.95 nuevos soles. 


\section{Cuadro 7}

\section{Costos variables por cerdo por tratamiento}

\begin{tabular}{|c|c|c|c|}
\hline \multirow[t]{2}{*}{ Variables } & \multicolumn{2}{|c|}{ Tratamientos } & \multirow[b]{2}{*}{ T3 } \\
\hline & $\mathrm{T} 1$ & T2 & \\
\hline $\begin{array}{l}\text { Consumo de alimento } \\
\text { ( } \mathrm{Kg} \text { /animal) }\end{array}$ & 116.31 & 89.78 & 85.58 \\
\hline $\begin{array}{l}\text { Costo del alimento } \\
(\mathrm{S} / \mathrm{kg})\end{array}$ & 0.234 & 0.221 & 0.207 \\
\hline $\begin{array}{l}\text { Costo total del alimento } \\
\text { (SI.) }\end{array}$ & 27.22 & 1984 & 17.72 \\
\hline
\end{tabular}

\section{Cuadro 8}

\section{Costo fijo por cerdo por tratamiento}

\begin{tabular}{lr}
\hline Variables & $\mathrm{S} /$. \\
\hline & \\
Gorrino & 21.49 \\
Sanidad & 1.50 \\
M ano de obra & 1.50 \\
Depreciación de inst. y equipos & 0.25 \\
\hline Total & 24.49
\end{tabular}

El análisis económico se muestra en el cuadro 9, donde se observa una utilidad neta por kilo de carne en nuevos soles de $0.210 ; 0.085$ y -0.056 para los tratamientos de $\mathrm{T} 1, \mathrm{~T} 2$ y $\mathrm{T} 3$, respectivamente. El mejor beneficios del T1 (testigo), a pesar de ser una ración más costosa, que el T2 y T3 se debió a la mayor ganancia de peso y una mejor conversión alimenticia encontrada en este tratamiento. 


\section{Cuadro 9}

\section{Beneficio promedio neto por animal por tratamiento}

\begin{tabular}{llllllcl}
\hline Trat. & P & Y (kg) & PxY & CV & CF & \multicolumn{2}{c}{ Beneficio Neto } \\
& & & & & & A nimal & K g carne \\
1 & 1.30 & 47.03 & 61.14 & 27.22 & 24.49 & 9.43 & 0.201 \\
2 & 1.30 & 36.50 & 47.45 & 19.84 & 24.49 & 3.12 & 0.085 \\
3 & 1.30 & 31.14 & 40.48 & 17.72 & 24.49 & -1.73 & -0.056 \\
\hline
\end{tabular}

$\mathrm{P} \quad=$ Precio por kilo de cerdo en nuevos soles.

$\mathrm{Y}=$ Peso promedio de cerdo por tratamiento en $\mathrm{kg}$.

$\mathrm{CV}=$ Costo variable de cerdo por tratamiento en nuevos soles.

$\mathrm{CF}=$ Costo fijo de cerdo por tratamiento en nuevos soles.

\section{CONCLUSIONES}

De los resultados obtenidos en el presente estudio se derivan las siguientes conclusiones

- Niveles mayores de $15 \%$ de harina de kudzu en la ración afecta el consumo de alimento y ganancia de peso de los cerdos en crecimiento.

- La mejor conversión alimenticia se obtuvo en el tratamiento con $0 \%$ de kudzu en la ración, seguidos por las raciones con $15 \%$ y $30 \%$ de harina de kudzu en la ración respectivamente.

- El mayor beneficio económico se logró en el tratamiento con 0\% de kudzu en la ración.

\section{BIBLIOGRAFIA}

BUITRAGO, J.A. 1990. La yuca en la alimentación animal. Centro Internacional de A gricultura Tropical. Cali, Colombia. 446 p.

CALZADA， J. 1982. M étodos estadísticos para la investigación 5ta. Edición, Lima, Perú, Editorial Milagros S.A. 644 p.

CASTILL O, S. 1992. Utilización de insumos no tradicionales en la alimentación de cerdos en la fase de crecimiento en el trópico. U niversidad $\mathrm{N}$ acional A graria de la Selva. Tingo M aría, Perú. 70p. 
COCHRANE, 1.1. 1982. "Caracterización agroecológica para el desarrollo de pasturas en suelos ácidos en A mérica tropical. En: Toledo, .1.M. (ed); Manual para la Evaluación Agronómica, Red Internacional de Pastos Tropicales, Centro Internacional de Agricultura Tropical. Cali, Colombia. pp. 2344

CONCELLON A.M. 1983. Porcinocultura. Barcelona, España. Editorial AED OS, T2, 101-103 pp.

ENSM INGER, M.E. y OLENTINE, C.G. 1983. A limentos y nutrición de los animales. Buenos A ires, A rgentina. Editorial El A teneo. $682 \mathrm{p}$.

HARRIS, J. 1988. International Feed nomenclatura and methods for summerizing and using, feed-data to calculate diet, st. Bult selt, $100 \mathrm{p}$.

HARRY, M. 1984. Utilización de forrajes como alimento de monogástricos. Instituto de Investigación A gropecuaria de Panamá. Panamá 29 p.

LUCAS, H. L. 1986. Pornicultura. 5ta. edición. Barcelona. España. Editorial AEDOS. Ti. 241 p.

MAYNARD, L.A. et al 1989. Nutrición Animal 7ma. Edición. México, Editorial M C GRAW-HILL. 640 p.

REYES, C. y ORDOÑEZ, H 1985. "Establecimiento y producción de leguminosas forrajeras en Pucallpa, Perú". En : Pizarro, E.A. (ed). Reunión de la Red Internacional de Evaluación de Pastos Tropicales. 3ra. Cali, Colombia. Resultados 1982-4985. Centro Internacional de A gricultura Tropical, Cali, Colombia, V.1 p. 647-656.

ROJAS, S.W. 1982. Nutrición animal aplicada. A ves, porcinos y vacunos. Departamento Académico de Nutrición y Escuela de Post Grado. U niversidad Nacional A graria, La M olina, Lima, Perú. 255 p.

RUIL OBA, 5. 1990. Efecto de la suplementación energética en invierno sobre la producción de leche, en base a Brachioaria decumbens y un banco de kudzu. Instituto de Investigación A gropecuaria de Panamá (IDIA P). Edición 12. $8 \mathrm{p}$. 
SAROLI, A.N. 1983. Efecto de diferentes niveles de harina de kudzu (Pueraria phaseoloides) en raciones de crecimiento y engorde de cerdos. Tesis de Ing. Zootecnista. U niversidad A graria de la Selva. Tingo M aría, Perú 68 p.

SONAIYA, E.B.; OMOLE, T.A.; ADEGBOLA, H.A. 1982. Effects of methionine suplemented cassava meal diets on performance and carcass characteristics and sorne organ weights of growing finishing pigs. Nutrition Report International $26(1): 25-34$. 